\title{
Coronavirus Disease 2019 (COVID-19) Associated With Febrile Status Epilepticus in a Child
}

\author{
Madhuradhar Chegondi ${ }^{1}$, Harsh Kothari ${ }^{2}$, Swathi Chacham ${ }^{3}$, Aditya Badheka ${ }^{4,5}$ \\ 1. Pediatrics, University of Iowa Stead Family Children's Hospital, Iowa City, USA 2. Division of Pediatric Critical Care \\ Medicine, Stead Family Children's Hospital, Iowa City, USA 3. Pediatrics, All India Institute of Medical Sciences, \\ Rishikesh, IND 4. Pediatrics, University of Iowa Hospitals and Clinics, Iowa City, USA 5. Pediatrics, Stead Family \\ Children's Hospital, Iowa City, USA
}

Corresponding author: Madhuradhar Chegondi, madhuradhar-chegondi@uiowa.edu

\begin{abstract}
Infection associated with the novel severe acute respiratory syndrome coronavirus 2 (SARS-CoV-2) has been named coronavirus disease 2019 (COVID-19). The emerging literature suggests that SARS-CoV-2 infection affects children of all age groups. COVID-19 as a cause of febrile seizures and status epilepticus is not yet reported in children. We report the case of a two-year-old child who presented to our pediatric intensive care unit with febrile status epilepticus and was diagnosed to have COVID-19 infection. The child recovered fully and was discharged home after three days.
\end{abstract}

Categories: Neurology, Pediatrics, Infectious Disease

Keywords: covid-19, sars-cov-2, febrile status epilepticus, child

\section{Introduction}

The novel severe acute respiratory syndrome coronavirus 2 (SARS-CoV-2) emerged in Wuhan City, Hubei Province, China, in December 2019. Infection associated with it was named Coronavirus disease 2019 (COVID-19). Ever since the first case was identified, COVID-19 rampantly spread all over the world and resulted in more than 15 million cases and 619,000 deaths globally [1]. The emerging literature suggests that the SARS-CoV-2 infection can affect children, including all age groups, predominantly males, and cause milder disease compared to adult patients [2,3]. In the United States, $1.7 \%$ of all confirmed COVID-19 cases are children ( $\leqslant 18$ years), and $5 \%-20 \%$ of them were hospitalized [4]. Recently, two pediatric COVID-19 cases were reported with afebrile seizures [5,6]. However, COVID-19 as a cause of febrile seizures and status epilepticus has not yet been reported. We report the case of a two-year-old child who presented to our pediatric intensive care unit (PICU) with febrile status epilepticus and was diagnosed to have COVID-19 infection.

Received 07/22/2020 Review began $07 / 31 / 2020$ Review ended 08/10/2020 Published 08/18/2020

\section{() Copyright 2020}

Chegondi et al. This is an open access article distributed under the terms of the Creative Commons Attribution License CC-BY 4.0., which permits unrestricted use, distribution, and reproduction in any medium, provided the original author and source are credited.

\section{Case Presentation}

A two-year-old, previously healthy female was transferred from a community hospital emergency department (ED) to our PICU with new-onset febrile seizure that presented as status epilepticus. The child started with a tactile fever and reduced oral intake a few hours earlier on the day of admission. Her parents took her to the local ED, where she was noted to have a temperature of $39.9^{\circ} \mathrm{C}$ with generalized tonic-clonic seizures. An intravenous (IV) access was established, and the patient received a dose of lorazepam. Furthermore, she was given four more doses of lorazepam and levetiracetam to abort seizures. After that she was noted to have respiratory depression and oxygen desaturation. She was endotracheally intubated with propofol and rocuronium and placed on a mechanical ventilator. Given the ongoing COVID-19 pandemic, appropriate personal protective measures were taken during the endotracheal intubation. The patient also received a dose of IV acetaminophen and one normal saline bolus $20 \mathrm{~mL} / \mathrm{kg}$ for borderline blood pressure post-intubation. Infectious workup was pursued in the setting of febrile seizure, and screening labs were obtained. Blood culture and urine culture were collected, and a dose of ceftriaxone $50 \mathrm{mg} / \mathrm{kg}$ was given. Ethanol, salicylate, and acetaminophen levels were normal. Her urine drug screen was negative. Her complete blood count showed leukocytosis with a white cell count of $17.69 \times 10^{9} / \mu \mathrm{L}$, neutrophil predominance, and microcytic anemia. She had no thrombocytopenia or lymphopenia. Her C-reactive protein was mildly elevated at $1.4 \mathrm{mg} / \mathrm{dL}$; her electrolytes, blood glucose, urea, creatinine, and liver function test were unremarkable. Her lactic acid was elevated at $5 \mathrm{mmol} / \mathrm{L}$. The urine analysis showed mild ketones and protein without evidence of urinary tract infection. The respiratory viral panel was negative. COVID-19 testing was sent, and the result was pending. A CT image of the brain was normal. Per the parent report, the child is fully immunized and did not have nasal congestion, cough, or gastrointestinal symptoms. The parents denied seizures or COVID-19 exposure in the family. However, her father had cough and congestion for the previous few days and was not tested for COVID-19.

The patient was transferred to our PICU on propofol infusion. Upon arrival to PICU, she was appropriately sedated on the mechanical ventilator, and was normothermic, with stable hemodynamics. The child was able to move all extremities in deep stimulation, and her bilateral pupils were equal and reactive to light. 


\section{Cureus}

Admission blood gas and repeat lactate were normal, and her chest X-ray showed patchy infiltrates bilaterally (Figure 1).

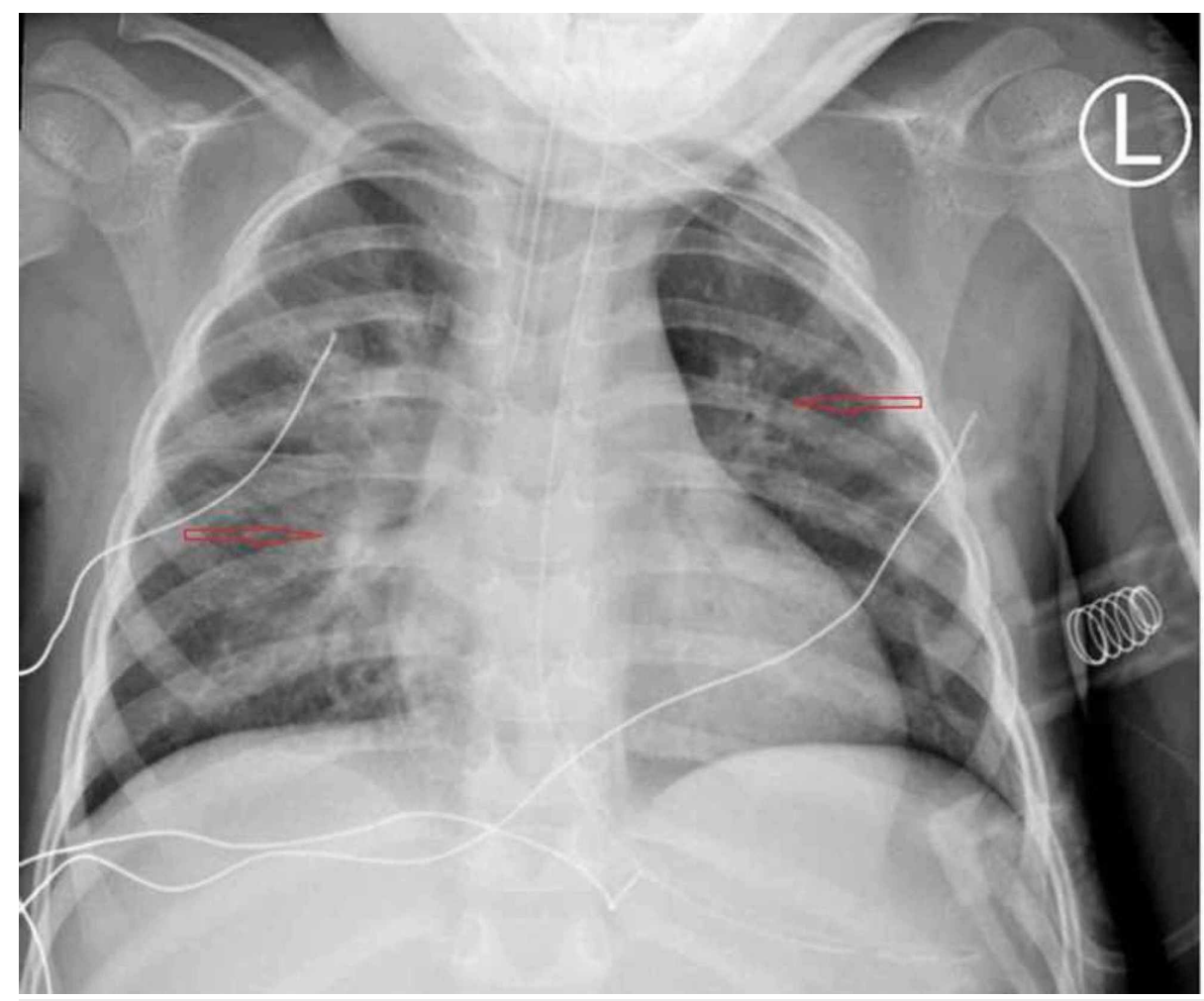

FIGURE 1: Chest X-ray showing bilateral patchy atelactasis

A repeat nasopharyngeal swab using real-time polymerase chain reaction for COVID-19 was sent and returned positive. Within two hours following admission, the child had a recurrence of seizure, which was aborted with a dose of lorazepam and a loading dose of fosphenytoin. The neurology service was consulted, and the patient was started on maintenance levetiracetam. The child was kept on continuous electroencephalogram (CEEG) monitoring for 24 hours. CEEG showed generalized slowing without recurrence of seizures. A lumbar puncture was performed, and cerebrospinal fluid (CSF) was sent for analysis. CSF showed one nucleated cell per $\mathrm{mm}^{3}$, less than 500 red blood cells per $\mathrm{mm}^{3}$, protein $12 \mathrm{mg} / \mathrm{dL}$, and glucose $86 \mathrm{mg} / \mathrm{dL}$. The CSF viral meningitis and encephalitis panel was negative. Due to testing restrictions, we were unable to send for COVID-19 testing in CSF or serology. The patient was continued on ceftriaxone, and vancomycin was added. On day 2 of the PICU stay, she was extubated under deep sedation to nasal cannula oxygen, and then was gradually weaned off to ambient air. She remained afebrile throughout her PICU stay, and antibiotics were discontinued as 48-hour cultures were negative. The child was discharged the following day on levetiracetam maintenance and advised to follow up with the neurology outpatient clinic in two weeks.

\section{Discussion}

The SARS-CoV-2 virus is one of the beta coronaviruses, which also includes severe acute respiratory syndrome virus (SARS-CoV), the Middle Eastern respiratory syndrome (MERS) virus, HCoV-HKU1, and HCoV-OC43 [7]. Alpha coronaviruses include HCoV-229E and HCoV-NL63 [7]. Among human coronaviruses (HCoVs), HCoV-229E, -OC43, -NL63, and -HKU1 strains are endemic worldwide [8]. HCoVs mostly cause upper respiratory tract illness (URTI) and can cause severe lower respiratory tract illness in children with underlying comorbidities. However, in COVID-19 cases, URTI is less frequent [9]. Among pediatric COVID19 cases in the United States, fever and cough present in $56 \%$ and $54 \%$ of the cases, respectively, and intensive care admission was required in $1.8 \%$ of them $[2,4]$.

In children, the extrapulmonary manifestations of $\mathrm{HCoV}$ include acute gastroenteritis, myocarditis, and multi-organ failure [8]. HCoV-associated neurological features include meningitis, encephalitis, acute flaccid paralysis, acute disseminated encephalomyelitis, and refractory status epilepticus $[8,10]$. Among the coronaviruses, HCoV-HKU1 has been reported as a common cause of febrile seizures in children [11]. Neurological involvement in COVID-19 patients has been described. A retrospective study from China reported that common neurological symptoms in adult patients with COVID-19 include headache, dizziness, and rarely seizures [12]. Karimi et al. reported frequent seizures in a young adult with COVID-19 [13]. In 
pediatric COVID-19 cases, headache is less frequently reported compared to adult patients [4]. However, as we were writing this case report, we could not find febrile seizures in children that were associated with COVID-19, though afebrile seizures were reported [5,6]. The possible mechanisms of coronavirus-associated neurological manifestations are direct virus invasion and effect of inflammatory mediators [10]. A study by Huang et al. reported that COVID-19 is associated with cytokine storm, and these cytokines might result in neuronal excitability and seizures [9]. It has been shown that HCoV-O43,-229E, and SARS-CoV are neuroinvasive [8,14]. Fatal encephalitis has been described with HCoV-OC43 in immunocompromised children $[15,16]$. In these children, the CSF analysis was negative, but the brain biopsy RNA sequencing was positive for $\mathrm{HCoV}-\mathrm{OC} 43$. However, in our index case, CSF was normal, and rapid improvement in sensorium suggests that COVID-19-associated encephalitis is unlikely. Due to limited COVID-19 testing capability, we could not check the CSF for SARS-CoV-2.

\section{Conclusions}

In children, COVID-19 incidence is lower, and severe disease is rare compared to adults. The majority of children with COVID-19 present with cough and fever. Febrile seizures have been described with HCoV infections in children, especially with HCoV-HKU1. Our index case illustrates that SARS-CoV-2 associated COVID-19 can present with febrile seizure and febrile status epilepticus in children.

\section{Additional Information \\ Disclosures}

Human subjects: Consent was obtained by all participants in this study. Conflicts of interest: In compliance with the ICMJE uniform disclosure form, all authors declare the following: Payment/services info: All authors have declared that no financial support was received from any organization for the submitted work. Financial relationships: All authors have declared that they have no financial relationships at present or within the previous three years with any organizations that might have an interest in the submitted work. Other relationships: All authors have declared that there are no other relationships or activities that could appear to have influenced the submitted work.

\section{References}

1. Johns Hopkins Coronavirus COVID-19 Resource Center. COVID-19 data in motion . (2020). Accessed: July 22, 2020: https://coronavirus.jhu.edu/ .

2. Lu X, Zhang L, Du H, et al.; Chinese Pediatric Novel Coronavirus Study Team: SARS-CoV-2 infection in children. N Engl J Med. 2020, 382:1663-1665. 10.1056/NEJMc2005073

3. Dong Y, Mo X, Hu Y, Qi X, Jiang F, Jiang Z, Tong S: Epidemiology of COVID-19 among children in China . Pediatrics. 2020, 145:e20200702. 10.1542/peds.2020-0702

4. CDC COVID-19 Response Team: Coronavirus Disease 2019 in children- United States, February 12-April 2 2020. MMWR Morb Mortal Wkly Rep. 2020, 69:422-426. 10.15585/mmwr.mm6914e4

5. Farley M, Zuberi J: COVID-19 precipitating status epilepticus in a pediatric patient . Am J Case Rep. 2020, 21:e925776.

6. Bhatta S, Sayed A, Ranabhat B, Bhatta RK, Acharya Y: New-onset seizure as the only presentation in a child with COVID-19. Cureus. 2020, 12:e8820. 10.7759/cureus.8820

7. International Committee on Taxonomy of Viruses . (2020). Accessed: June 24, 2020: https://talk.ictvonline.org/.

8. Desforges M, Le Coupanec A, Dubeau P, Bourgouin A, Lajoie L, Dubé M, Talbot PJ: Human coronaviruses and other respiratory viruses: underestimated opportunistic pathogens of the central nervous system? . Viruses. 2019, 12:14. 10.3390/v12010014

9. Huang C, Wang Y, Li X, et al.: Clinical features of patients infected with 2019 novel coronavirus in Wuhan, China. Lancet. 2020, 395:497-506. 10.1016/S0140-6736(20)30183-5

10. Robinson CP, Busl KM: Neurologic manifestations of severe respiratory viral contagions . Crit Care Explor. 2020, 2:e0107. 10.1097/CCE.0000000000000107

11. Lau SK, Woo PC, Yip CC, et al.: Coronavirus HKU1 and other coronavirus infections in Hong Kong. J Clin Microbiol. 2006, 44:2063-2071. 10.1128/JCM.02614-05

12. Mao L, Jin H, Wang M, et al.: Neurological manifestations of hospitalized patients with COVID-19 in Wuhan, China. JAMA Neurol. 2020, 77:683-690. 10.1001/jamaneurol.2020.1127

13. Karimi N, Sharifi Razavi A, Rouhani N: Frequent convulsive seizures in an adult patient with COVID- 19: a case report. Iran Red Crescent Med J. 2020, 22:e102828. 10.5812/ircmj.102828

14. Principi N, Bosis S, Esposito S: Effects of coronavirus infections in children . Emerg Infect Dis. 2010, 16:183188. 10.3201/eid1602.090469

15. Nilsson A, Edner N, Albert J, Ternhag A: Fatal encephalitis associated with coronavirus OC43 in an immunocompromised child. Infect Dis. 2020, 52:419-422. 10.1080/23744235.2020.1729403

16. Morfopoulou S, Brown JR, Davies EG, et al.: Human coronavirus OC43 associated with fatal encephalitis . N Engl J Med. 2016, 375:497-498. 10.1056/NEJMc1509458 